\title{
La teología latinoamericana ante los retos epocales
}

\author{
Pedro Trigo, \\ Centro Gumilla, \\ Caracas
}

Para analizar la teología que se produce en América Latina hay que comenzar haciéndose cargo del momento que vive la región, porque el cristianismo, con su talante de encarnación kenótica ${ }^{1}$, entraña, en términos de Ellacuría, hacerse cargo de la realidad, encargarse de la realidad y cargar con ella ${ }^{2}$, a lo que Sobrino, en consonancia con la gracia que está al origen de la Encarnación, añadió el ser

1. "Encarnación" es una palabra mayor que expresa la sensibilidad y la dirección vital que caracterizaron al Concilio Vaticano II y a quienes participan de su espiritualidad. Ver P. Trigo, Espiritualidad conciliar, Universidad Iberoamericana de Puebla/ ITESO, 2003, pp. 76-99; J. M. Mardones, "Del catolicismo tradicional al conciliar: del sagrado poseído al encarnado", en La transformación de la religión, PPC, Madrid, 2005, pp. 139-152,161-162, 189-193. Sobre la kénosis de Jesús y la de Dios, ver J. I. González Faus, La humanidad nueva, ST, Santander, 1984, pp. 195-214; J. Moingt, El hombre que venía de Dios, vol. II, DDB, Bilbao, 1995, pp. 209-251; J. Sobrino, Jesucristo Liberador, Trotta, Madrid, 1991, pp. 297-320; J. Sobrino, La fe en Jesucristo, Trotta, Madrid, 1999, pp. 363-387. Una fenomenología de la encarnación para comprender el misterio de la Encarnación y el misterio como caso límite de la fenomenología, véase M. Henry, Encarnación, Sígueme, Salamanca, 2001.

2. I. Ellacuría, "Hacia una fundamentación del método teológico latinoamericano", en Escritos teológicos, I, UCA Editores, San Salvador, 2000, p. 208. Son estas expresiones frecuentes en Sobrino. Ver, por ejemplo, “"El pueblo crucificado' y 'la civilización de la pobreza'. 'El hacerse cargo de la realidad' de Ignacio Ellacuría, $R L T, 66$ (2005), pp. 209-228; "La interpelación a 'ser real", en La fe en Jesucristo, op. cit., pp. 405-409. 
cargados por ella ${ }^{3}$. Así, pues, una teología cristiana responsable es siempre una teología de la realidad ${ }^{4}$ y desde la realidad ${ }^{5}$.

Pero es que además hoy, por razones en parte distintas a las de los años sesenta y setenta, es especialmente difícil vivir en la realidad en América Latina porque, al estar abriéndonos a una época nueva, tanto en la región como a nivel mundial, no es fácil hacerle justicia, tanto por la inercia que lleva a permanecer en lo que se vivió, máxime si tuvo relevancia, aunque fuera a un alto precio, como porque no es tan fácil discernir entre los bienes civilizatorios y culturales de la época que se abre y la dirección dominante que hasta hoy prevalece en ella, que, por lo que toca a la globalización, configura un totalitarismo de mercado, y por lo que toca a América Latina, se caracteriza por la cerrazón de las élites a lo que puja por venir y la poca claridad de bastantes que lideran lo nuevo.

\section{Teología y discernimiento de la globalización desde América Latina}

\subsection{La falta de relevancia, si no hay pretensión trascendente, origina una teología enclaustrada: academicismo}

El teólogo latinoamericano tiene que hacerse cargo de la nueva revolución de la informática y, más todavía, de la genética, así como de la globalización del comercio, la producción y las finanzas, de los flujos migratorios, de los organismos multinacionales; cambios que permiten la presencia de todos a todos y de modo incipiente, pero cada vez más acelerado, la influencia mutua y la interacción. Asumir esas innovaciones y ese cambio de escala, con las transformaciones personales consiguientes, consume mucho tiempo y energías, más aún cuando esos cambios no han salido de nuestra región.

Pero dificulta más asumirlos porque es imprescindible discernir entre esos bienes y el dominio brutal e infecundo de las grandes corporaciones y, en definitiva, de los grandes inversionistas, que desestructuran a las personas para volverlas adictas a sus productos y supeditan todo a sus ganancias y, que, para hacerlo en democracia, provocan una gigantesca ideologización que encubre la realidad.

3. J. Sobrino, "La centralidad del reino de Dios anunciado por Jesús", RLT, 68 (2006), pp. 157-159.

4. J. Sobrino, "Teología desde la realidad", en L. C. Susin (ed.), El mar se abrió, Sal Terrae, Santander, 2001, pp. 140-156; J. Comblin, La oración de Jesús: asumir la densidad del mundo desde Dios,Sal Terrae, Santander, 1977.

5. Una síntesis programática de los cambios que han acontecido en la sociedad y en la Iglesia y una toma de posición lúcida de la misión de la teología a lo largo del proceso se halla en Ensino e método da teologia, Perspectiva Teológica, 31 (1999), pp. 5-10. 
No es fácil situarse en esta realidad histórica y discernirla cuando las élites de nuestros países buscan, al costo que sea, subirse apendicularmente al carro de la globalización, cuando las instituciones universitarias de la región luchan por funcionalizarse y las facultades de teología sienten la presión de cumplir esos estándares a nivel formal, una presión tan apremiante que, si no se pone mucho cuidado, roba las energías y más aún el interés y la dirección vital de los teólogos académicos.

Así, pues, el teólogo latinoamericano se siente periférico respecto de la dirección dominante mundial y de los requerimientos de las élites locales, globalizadas o en lucha por entrar en la globalización. Sabiendo que no interesa lo que hace, el teólogo universitario tiende a integrarse al establecimiento por el cumplimiento de los requerimientos formales, con lo que consigue subir de rango y obtener reconocimiento y financiamiento estatal. Esto se da en el marco de una teología académica cada vez más absorbida por dar la talla entre las instituciones de élite. De este modo, el análisis de textos y autores, y más en general la historia de las ideas y, por lo que toca a Jesús y a la Iglesia, el análisis histórico, van dando la pauta, orillándose cada vez más el elemento que constituye a la teología cristiana: la fe, que es la que busca entender, porque la teología es intelectus fidei $^{6}$, amoris $^{7}$, gratiae, misericordiae, liberationis ${ }^{8}$.

Este tipo de teología academicista pasa de largo ante el totalitarismo de mercado, que configura no solo una situación de pecado, sino un misterio de iniquidad, y si lo alude, lo hace neutramente, como hablan los dueños de este mundo y sus intelectuales, que se refieren a la flexibilización del mercado de trabajo, a alentar a los inversores, a dar confianza a los mercados, a la sociedad del riesgo, como antes se refirieron en América Latina a las entradas, a la pacificación, a los repartimientos y encomiendas, y más en general a los bienes que trajeron los europeos a los indígenas, muy superiores, según ellos, al oro y la plata que les quitaron ${ }^{9}$.

6. Susin lo sintetiza satisfactoriamente, aunque no explicita que esa revelación acontece históricamente, lo que entraña consecuencias metodológicas. Ver L. C. Susin, "Fazer teologia em tempos de globalizaçâo", Perspectiva Teológica, 31 (1999), pp. 98-101.

7. Para las consecuencias concretísimas de entender así la teología, ver J. Martínez Gordo, “Dios en prisión?”, RLT, 83 (2011), pp. 249-254.

8. J. Sobrino, El principio misericordia, Sal Terrae, Santander, 1992, pp. 65-80; y "Teología desde la realidad", op. cit., p. 147.

9. Es pertinente recordar que la primera vez que se expuso y defendió sistemáticamente el colonialismo fue en una disputa teológica, en la que, por cierto, se rebatió también, acudiendo precisamente al Evangelio. Ver Á. Losada, Apología de Juan Ginés de Sepúlveda contra fray Bartolomé de las Casas, Editora Nacional, Madrid, 1975, pp. 76 y 134-138. 
También, la teología fundamentalista y doctrinaria, en auge en nuestro continente, aupada por los nuevos movimientos y por una parte de la jerarquía, ha asumido este ropaje académico formal y dedica trabajos eruditos a cuestiones del pasado, sin apenas hermenéutica o como revestimiento de cuestiones doctrinarias, tratadas con espíritu premoderno. Una teología en general que, por su carácter anacrónico, no contiene inteligibilidad.

Así, pues, la adaptación formalista a la época globalizada oscila entre la instalación intrascendente en lo establecido, ya que esos teólogos dicen lo que podría decir un científico del establecimiento, y la proclamación insignificante, ya que lo que se propone doctrinariamente no solo no tiene sentido, sino que carece de significado para quien no pertenezca a la secta, en el sentido sociológico de colectivo cerrado.

Esa falta de significatividad por lo que respecta al contenido se duplica por el formato académico no solo en el sentido del lenguaje que se emplea, que resulta ininteligible para el pueblo de Dios y los ciudadanos en general, sino más radicalmente porque no es un lenguaje performativo, no hay en él pretensión de servicio a la comunidad cristiana ni a gente abierta de buena voluntad. Es un ejercicio académico hecho, ciertamente, con buena voluntad y, se supone, con fe, pero fundamentalmente para cumplir requisitos académicos y teniendo, en el mejor de los casos, a académicos como interlocutores o como servicio escolar a los alumnos.

\subsection{La dificultad de una teología que sea profecía razonada y evangélica}

Esta situación mundial demanda desde la periferia una teología profética. Para hacerla se requieren dos condiciones: asumir la época, en cuanto a sus bienes civilizatorios y culturales, y rechazar la dirección dominante no solo ideológica, sino prácticamente, desde el compromiso solidario con las víctimas y el cuidado por la humanización de los de arriba, participando de la actitud del Dios revelado en Jesucristo. Esto se traduce en una profecía razonable y razonada, es decir, realmente trascendente y no asimilable por el establecimiento, más aún, condenatoria de él; pero dicha no desde el pasado, sino desde el futuro de Dios y en los cauces de la época, y dicha, en definitiva, como Evangelio ${ }^{10}$ para todos, por supuesto, los oprimidos y excluidos, pero incluidos también los dominadores, a los que se pide, ciertamente, conversión a esa buena noticia, que es costosa sin duda, pero que es Evangelio. Para que lo sea se requiere tanto que proponga a Dios concretamente como vida de nuestra vida y el seguimiento concreto de Jesús como camino de humanización, como que proponga cauces

10. Sobrino insiste frecuentemente sobre la condición de evangelio que debe tener toda teología que quiera ser legítimamente cristiana. Véase J. Sobrino, Jesucristo liberador, Trotta, Madrid, 1991, pp. 108-110. 
concretos de esta vida en seguimiento, cosa solo posible desde un amor concreto por las víctimas y, de otro modo, por los opresores ${ }^{11}$.

Unos teólogos se han visto tan implicados en las luchas populares de fines de los años sesenta hasta los noventa y se sienten tan derrotados y excluidos por los amos de hoy, que tienden a persistir no solo en sus solidaridades, lo que es legítimo, incluso indispensable, sino en el horizonte de la época pasada, con lo que lo que dicen, aun siendo verdadero y justo, no es el lenguaje de un contemporáneo a sus contemporáneos, y por eso estos lo pueden dejar de lado sin sentirse interpelados, porque captan que no les están hablando a ellos.

La profecía tiene que estar dirigida a los de su época y para eso hay que asumir el estado en que se encuentran las personas, de manera que ellas puedan comprender que la condena a la dirección dominante de esta figura histórica es en definitiva para que ellas tengan más posibilidades de vida y una vida más humana.

Otros, que en épocas anteriores pasaron de largo ante estos problemas tan acuciantes, o que se formaron sin asumirse dentro de ese Evangelio solidario, intentan decir algo, que ellos estiman pertinente y concreto, alejado de esas zonas conflictivas, pero que, al eludir la realidad de fondo, no es en verdad concreto, sino particular, y por eso, aunque contenga aportes, en definitiva, resulta poco trascendente.

Otros se atienen a la esfera religiosa de la realidad, aunque, como dijimos, la revisten de un manto de erudición. Estas personas viven ajenas a la profecía y, por ello, también al Evangelio ${ }^{12}$, ya que el Evangelio pasa de un modo u otro por la cruz, que es el destino del seguimiento de Jesús de Nazaret. Sin embargo, no sienten su insignificancia porque hoy, a diferencia de antaño, existe un discurso religioso establecido, y por eso tienen audiencia y recursos.

Creemos que una dirección de la teología latinoamericana que, a la vez que asuma esta época mundializada, tome postura contra su dirección dominante, totalitaria y fetichista, tiene que sentirse haciendo cuerpo con los teólogos de los otros terceros mundos que escriben desde esta misma perspectiva y no menos con los teólogos que la viven desde el Primer Mundo. Esto demanda un gran esfuerzo y muy concretamente abrir canales de comunicación, lo que está fuera

11. Esta doble exigencia está presente, p. ej., en G. Gutiérrez, "La opción por los pobres hoy”, RLT, 80 (2010), pp. 161-174; y en M. Eckholt, “Con pasión y compasión', movimientos de búsqueda de teólogas latinoamericanas”, Teología y Vida, vol. 48, n. 1 (2007), pp. 9-24.

12. Para la contraposición entre religión y Evangelio o, en el fondo, el dar prioridad a la religión sobre el Evangelio, a través de la historia del cristianismo hasta hoy y la alternativa de priorizar el Evangelio, ver J. Comblin, "Fe y política", RLT, 80 (2010), pp. 195-204. 
del alcance de la mayoría de los teólogos y ajeno al interés de la mayoría de las facultades de teología de la región.

La poca sensibilidad hacia un vínculo tan indispensable para mantener la trascendencia dinámica respecto del establecimiento en esta época mundializada es expresión elocuente o de la pobreza intelectual de las facultades o de su instalación. Creemos además que la mayoría de lo poco que se hace está inscrito en una postura, característica de la Ilustración, de lograr una suerte de religión mundial o un parámetro para medirlas a todas, en vez de dialogar fraternamente desde la fe de cada uno y por tanto desde el misterio que trasciende a todos los interlocutores y que ellos no intentan de ningún modo dominar.

\subsection{Discernir entre la religión neolítica y el cristianismo para que el fin de la primera no acarree el del segundo}

Todavía hay un punto que, a mi modo de ver, caracteriza a esta época y que tiene que ser visto y asumido por la teología. Esta época sería la superación de la que se abrió con el neolítico. Por tanto, también va a pasar, y ya lo estamos viendo, la religión neolítica, caracterizada por la tríada: templos, sacerdotes y sacrificios.

En absoluto, el cristianismo no tendría que tener ningún problema al respecto porque en las fuentes cristianas no existe esta tríada. Esto es tan patente en los Evangelios que no hace falta demostrarlo, ya que ellos lo asumen explícitamente. Las escasas citas sobre el sacrificio en otros escritos tienen un carácter metafórico y propedéutico para que entendieran la propuesta cristiana quienes estaban habituados a esas categorías. Por ejemplo, cuando Pablo pide a los romanos que ofrezcan sus cuerpos en sacrificio, que ese es el culto espiritual que agrada a Dios, lo que les pide en concreto es que no se amolden al orden establecido, sino que renueven su mente para que puedan discernir qué es lo bueno, lo que agrada a Dios, lo consumado. Por eso, cuando en Antioquía se sale del ámbito de la religión judía y se predica a Jesús a los paganos, sin pasar por el Antiguo Testamento, la gente se asombra de ver en los convertidos a gente muy religiosa que no tenía religión, es decir, templos, sacerdotes ni sacrificios. Por eso, los llamaron cristianos, mesiánicos, ungidos, tanto porque hablaban siempre de Jesús, el Mesías o ungido (en griego jristós: Cristo), como porque ellos mismos se sentían ungidos por el mismo Espíritu de Jesús, su Señor.

Sin embargo, la presión de la religión neolítica fue tan fuerte que ya en el siglo III se empieza a hablar del orden sacerdotal, en unos, como Tertuliano, peyorativamente, y con razón, porque si los presbíteros y obispos entraban en ese escalafón, se imposibilitaba vivir la fraternidad en las comunidades cristianas. Sin embargo, desde Constantino, la Cena del Señor, centro indiscutible de la comunidad, pasó a hacer las veces del sacrificio, para la ciudad y el orbe, que 
sostenía al imperio: se transformó en un acto de culto no solo público, sino político. Por eso, aunque en un primer momento no se aceptaron los templos, las iglesias empezaron a ser vistas como tales y a asumir su función.

Se puede discutir si el resultado fue un cristianismo religioso, en el sentido de la religión del neolítico, inculturación ciertamente válida, o una religión neolítica con formas cristianas, aculturación reductora. Seguramente en unos tiempos, lugares y personas predominó lo cristiano y en otros lo religioso neolítico.

El problema para América Latina es que nació estructuralmente como religión cristiana, ya que la Iglesia colonial (no la de la etapa constituyente de los fundadores), como Iglesia postridentina que era, por reacción adialéctica a la reforma luterana, desconoció la Biblia y el sacerdocio de los fieles, y fue una Iglesia de doctrina, culto y disciplina, que tenía como sujeto a la clerecía y como destinatarios a los fieles.

Esa negativa a reconocer el carácter de sujeto de los fieles y sobre todo del pueblo fue lo que dio lugar a la religiosidad popular que, como reconoció Puebla, "no solamente es objeto de evangelización, sino que, en cuanto contiene encarnada la Palabra de Dios, es una forma activa con la cual el pueblo se evangeliza continuamente a sí mismo"13. Esa manera de interpretar y vivir el cristianismo, aunque tiene un importante anclaje ritual, se expresa sobre todo en la vida. En ella se rastrea la presencia de Dios y se responde a su acción. También la asunción del Vaticano II en Medellín supuso en principio reponer la primacía de lo cristiano y así se practicó eximiamente en comunidades populares y en grupos solidarios con ellas. En ellas, nuevamente la misa volvió a ser la Cena del Señor de las comunidades de los primeros siglos. La fe, la esperanza y la caridad se realizaban en la vida y se celebraban con gran alegría y creatividad en la Cena del Señor.

Sin embargo, al no aceptar las instancias centrales el Vaticano II, se ha presionado fortísimamente a volver a la tríada neolítica que relega a los cristianos a destinatarios de la acción cultual que realizan los sacerdotes.

No obstante, desde comienzo de siglo se va haciendo patente en nuestra región que ya no se transmite ambientalmente el cristianismo. Ante esta constatación, que llena de angustia a no pocos, una parte considerable de la institución eclesiástica se atrinchera en lo que considera el mínimo indispensable a defender, que, en la práctica, para ellos, no es el Evangelio, sino la tríada de templos, sacerdotes y sacrificios: la religión neolítica. En el fondo se están defendiendo a sí mismos, identificados con ese horizonte. Por eso, una parte considerable de la institución eclesiástica constituye la principal dificultad para que los cristianos

13. Puebla, n. 450 . 
latinoamericanos puedan desprenderse de ese revestimiento y afincarse en la novedad cristiana y, por tanto, vivir proactivamente en esta nueva época.

Hay aquí varios problemas para la teología. El primero, hacerse cargo de esta novedad y teorizarla adecuadamente, porque acecha un doble problema polar: unos teólogos tienden a confundir el fin de la religión neolítica con el fin de la religión y esto no es ni analíticamente acertado ni cristianamente válido. No es analíticamente acertado porque una característica que distingue la postmodernidad de la modernidad es precisamente el retorno de la religión ${ }^{14}$. Se podrá discutir sobre su ambigüedad, pero no sobre su presencia. No es tampoco cristianamente válido porque Jesús es el Hijo único de Dios que nos lo ha revelado, no en el sentido de desvelarnos algo que ya existía, pero estaba oculto para nosotros, sino como acontecimiento: al hacerse nuestro hermano, nos ha introducido en su relación filial. Si no hay religión, no tiene sentido que lleguemos a ser hijos de Dios en su Hijo único Jesús. Cierto es que Jesús revela, también como acontecimiento, en qué consiste el ser humano al propio ser humano ${ }^{15}$. Pero lo que revela es precisamente que es, en Jesús de Nazaret, hijo de Dios y hermano de todos, desde la primacía de los pobres. No podemos reducir el cristianismo a un humanismo, si no especificamos que es el de los hijos de Dios, referido, pues, no solo a todos como hermanos, sino a Dios como Padre común con entrañas de madre.

El otro peligro polar es el de identificar al cristianismo con ese revestimiento histórico neolítico, lo que implica absolutizarlo, con lo que de hecho se relativiza la novedad cristiana y además se unce el cristianismo a una manera de concebir y practicar la religión que tiene sus días contados. Esta falta radical de discernimiento es suicida, pero sobre todo no hace justicia a las fuentes y ladea los Evangelios y a Jesús de Nazaret y su seguimiento.

Así, pues, esta es una tarea epocal imprescindible de la teología, que no la podrá llevar a cabo si no sale de su enclaustramiento académico y no se entraña en la comunidad eclesial para que ella sienta que se le habla desde dentro, es decir, como un hermano en la $\mathrm{fe}^{16}$, y a la vez, desde las fuentes, desde la autoridad de las fuentes y no meramente desde la propia opinión de ilustrado. Si el teólogo no da ese paso, no podrá influir legítima y fecundamente en un tema tan sensible y decisivo.

14. J. M. Mardones, Síntomas de un retorno, Sal Terrae, Santander, 1999; J. B. Libanio, "O paradoxo do fenômeno religioso no início do milênio", Perspectiva Teológica, 34 (2002), pp. 63-88.

15. Sobre la revelación conjunta que Jesús de Nazaret hace al ser humano, tanto de Dios como del propio ser humano, ver Gaudium et spes 22.

16. L. C. Susin, "Fazer teologia em tempos de globalizaçâo", op. cit.; C. Palacio, "Da 'Humani generis' à 'Fides et ratio", Perspectiva Teológica, 31 (1999), pp. 35-43. 
En América Latina esto es especialmente decisivo porque al no transmitirse ya ambientalmente el cristianismo, es suicida inclinar a los cristianos a atrincherarse frente a esta época negándose a entrar en ella e insistiendo en lo acostumbrado (templos, sacerdotes y sacrificios), en vez de propiciar un encuentro personal con Jesús de Nazaret a través de los Evangelios, para que su vida quede religada a él como un verdadero seguidor y testigo.

\section{Discernimiento de la tercera época latinoamericana y teología}

Pero si para un teólogo latinoamericano no es fácil asumir la globalización de un modo alternativo a la dirección hasta ahora dominante, le es más difícil aún encarar desde el Espíritu de Jesucristo la tercera época que se está abriendo paso muy dificultosamente en nuestra América.

La primera fue la época amerindia, la segunda tuvo como protagonistas a los peninsulares, devenidos en el siglo XX en occidentales, a causa de las migraciones. En esta época tenemos que distinguir tres períodos: en el primero comparten el poder los españoles y lusitanos europeos, y los americanos; en el segundo, los americanos se emancipan de los europeos, pero para continuar la sociedad señorial; por eso, las repúblicas, sea cual sea la constitución, son señoriales. En el tercer período, que se impone en la segunda mitad del siglo pasado, los occidentales comparten el poder con todas las etnias, pero con la condición de que se occidentalicen. Los cauces de occidentalización fueron la educación, los partidos políticos y los medios de comunicación de masas. Es el período de modernización democrática. Pero las expectativas que se crearon en el pueblo se estrellaron contra el muro de las élites que no les quisieron dar paso. De ahí, los regímenes de la seguridad nacional o las democracias de fachada, como la mexicana.

En este proceso se jugó la Iglesia: la que recibió creativamente el Vaticano II en Medellín y todavía pudo expresarse en Puebla, a pesar del cerco eclesiástico y político. Era un proceso en base a la subjetualidad popular y las organizaciones de base. Un proceso replicado en la Iglesia con las comunidades eclesiales de base, la pastoral orgánica desde la base y la propuesta de un cambio de solidaridades desde la pobreza institucional. En Medellín nació la Iglesia latinoamericana, como cuerpo orgánico articulado y con una propuesta histórica de indudable raigambre evangélica, y también nació su teología ${ }^{17}$, que empató

17. J. Noemi, "El porvenir de los católicos latinoamericanos", Teología y Vida, vol. 48, n. 1 (2007), pp. 105-110. 
con la de los fundadores ${ }^{18}$ y con otras producciones más o menos aisladas, pero altamente significativas ${ }^{19}$.

Esta Iglesia de los pobres corrió la suerte de los pueblos. Pero estos se han repuesto de su derrota y exigen a todos entrar en la tercera época, que consiste en constituir una región multiétnica y pluricultural en un estado de justicia o, más exactamente, en que se reconozca ese carácter pluricultural en un estado de justicia e interacción simbiótica; es decir que nuestra América deje de ser exclusivamente latina en sus instituciones y símbolos, e incluya instituciones y símbolos de las demás culturas y a quienes viven en ellas, en pie de igualdad.

Esta es la demanda de indígenas, afrolatinoamericanos, campesinos y suburbanos, las cuatro culturas populares, a los de culturas dominantes: los de cultura occidental criolla, atrincherados en las instituciones, y los de cultura occidental mundializada. La prestancia es tal, que ellos han llevado a la presidencia a Chávez, a Morales, a Correa, a Lula (en la segunda vuelta en su segunda presidencia), a Lugo y probablemente también a los Kirchner y a Funes, y ellos lograron que López Obrador ganara las elecciones, aunque no se las reconocieron. No digo que esos presidentes representen genuinamente estas culturas, sino que ganaron porque se presentaron como abanderados de ellas y fueron reconocidos por sus cultores como tales.

¿Qué tiene que ver todo esto con la teología? En primer lugar, ella tiene que discernir el significado de esta época que se abre, en el doble sentido de aceptar que entramos en ella y de descubrir su carácter de kairós ${ }^{20}$. Para nosotros es claro que se trata de una oportunidad salvífica de tal calibre que hay que afirmar sin rodeos que quien se opone a ella se opone a $\operatorname{Dios}^{21}$.

18. La obra más eximia, una de las cumbres de la teología, fue, sin duda, De unico vocationis modo, de Las Casas. En Obras completas, 2, Alianza, Madrid, 1990.

19. Por ejemplo, las de los capuchinos Epifanio de Moirans, Siervos libres (Consejo Superior de Investigaciones Científicas, Madrid, 2007), y Francisco José de Jaca, Resolución sobre la libertad de los negros (CSIC, Madrid, 2002), contra la esclavitud de los negros; o El triunfo de la libertad sobre el despotismo, de Juan Germán Roscio (Monte Ávila, Caracas, 1983), autor del acta de independencia de Venezuela.

20. Sobre la necesidad de realizar este discernimiento y sobre cómo la teología latinoamericana se abocó a ello, ver J. Noemi, "En la búsqueda de una teología de los "signos de los tiempos", Teología y Vida, vol. 48, n. 4 (2007), pp. 439-447. Una síntesis de cómo lo hizo la teología de la liberación, en J. Costadoat, "Los 'signos de los tiempos’ en la Teología de la liberación”, Teología y Vida, vol. 48, n. 4 (2007), pp. 399-412. Un discernimiento ponderado y estimulante, en S. Silva, "La Teología de la Liberación", Teología y Vida, vol. 50, n. . 1-2 (2009), pp. 93-116.

21. P. Trigo, "Construir una América Latina pluricultural para contribuir proactivamente a una mundialización alternativa”, ITER Humanitas, 14 (2010), pp. 95-121. 
No es fácil para un teólogo latinoamericano hacer este discernimiento y mucho menos situarse como persona y como teólogo consecuentemente en la realidad de la hora. Para la mayoría, ocuparse del tema es considerado como distractivo, ya que su atención está dirigida a ser un buen académico y a hacer alguna contribución valiosa para la academia, con repercusión, si es posible, en Europa. Desde esa perspectiva, este planteamiento parece un provincianismo antihistórico, porque para él, la historia la marca la dirección dominante de esta figura histórica.

Además, si ya es difícil ser reconocido por la academia, mirar hacia las culturas populares demanda un cambio de horizonte, de referencias y de solidaridades. Y aquí viene el problema estrictamente personal, porque el teólogo promedio se define o como occidental americano o como occidental mundializado, y vive en pacífica posesión de esa identidad y, desde ella, le parece lógico que nuestra América siga siendo latina y no ve nada positivo en el multiculturalismo, si entraña, como así parece, algo más que se les deje vivir en sus culturas a la gente popular, sin dejar de empujar el proceso de modernización porque en definitiva se les hace un favor ayudándolos a que asuman la cultura occidental. Desde este planteamiento, la solidaridad con los de abajo pasa por su occidentalización integral, aunque todos estamos de acuerdo en que permanezcan rasgos culturales, recibidos por todos con aprecio como folclore. Desde este punto de vista, el teólogo está en su casa y los cristianos populares tienen que acabar de entrar en ella. Nosotros somos el paradigma que ellos tienen que investir. Nuestra América solo puede ser latina: una provincia de Occidente con algunos rasgos mestizos $^{22}$.

Esto es no abrirse a lo que el Espíritu impulsa en nuestra América y aquí estriba la mayor dificultad para hacer una teología genuina en América Latina.

Pero todavía hay mucho más. Si se entra por esta puerta, se deduce que la institución eclesiástica latinoamericana tiene que diversificarse: no puede seguir siendo solo occidental americana. Tiene que haber gente de barrio, campesinos, afrolatinoamericanos e indígenas curas y obispos, y se entiende que sin pasar por el seminario, que es un lugar de blanqueo. Pero para que eso sea posible con coherencia, hay que inculturar el cristianismo a las cuatro culturas populares y, en primer lugar, hay que reconocer la excelencia cristiana de no pocos cristianos de esas culturas. Ellos son los que tienen que inculturar. Lo que les corresponde a los occidentales es estimular su cristianismo.

22. Sobre el mestizaje como ideología y la utilización de símbolos cristianos que la avalen, para el caso, sintomático, de México, ver J. Gómez Izquierdo y M. E. Sánchez Díaz, La ideología mestizante, el guadalupanismo y sus repercusiones sociales, Universidad Iberoamericana de Puebla, 2011. 
Está claro que los teólogos tienen que participar de todo esto y que tienen que hacerlo entrando ellos en la casa del pueblo. Y de esta participación saldrán elaboraciones teológicas concretísimas; desde la densidad de la realidad y con el tiempo, teologías católicas en esas culturas. Ya existen muestras excelentísimas en muy diversas áreas ${ }^{23}$. Es una tarea hermosa, pero que supone un cambio tan grande en el mundo-de-vida de no pocos teólogos que equivale a volver a nacer, cosa imposible sin que sea captado como Evangelio de Dios.

\section{Conclusión abierta}

\subsection{Nuestra síntesis}

La profesionalización, entendida de modo prevalentemente formal, consume gran parte de las energías de los teólogos latinoamericanos y de las instituciones en las que laboran.

La teología viva en la región es la que se mide por el desafío del discernimiento de la época, tanto mundial como latinoamericana, desde el compromiso solidario con ella y más concretamente desde el lugar de las víctimas, porque solo cuando les vaya bien a los pobres les irá bien a todos, y desde los Evangelios y el seguimiento en creatividad fiel a Jesús de Nazaret en su Espíritu.

El discernimiento es en orden a una teología profética que no puede omitir la denuncia, pero que tiene que realizarse como Evangelio, dando una importancia relevante a la cotidianidad cualitativa según el paradigma de Jesús de Nazaret.

Esta teología encuentra serias dificultades, tanto en lo incipiente de las épocas, como en la constitución del propio teólogo, como en la dirección dominante a nivel mundial de nuestros países y eclesiástica. Pero se va dando.

\section{2. ¿Qué tiene que decir esta teología a la que se hace en España?}

Primero y principal, que en la época de la mundialización todos tenemos los mismos problemas y todos los que vamos en una dirección formamos un colectivo, que tiene que ir articulándose, lo que resulta particularmente indispensable para los que desde dentro de la época vamos a contracorriente en una dirección evangélica alternativa.

En segundo lugar, tenemos que decirnos todos que es una vergüenza que la teología no se aboque como tarea prioritaria a discernir la época mundializada en la que vivimos y proponer no solo el juicio cristiano, que entraña propuestas

23. V. Codina, Una Iglesia nazarena, Sal Terrae, Santander, 2011; R. Muñoz, Nueva conciencia cristiana en un mundo globalizado, LOM, Santiago de Chile, 2009; J. Mo Sung, Deseo, mercado y religión, Sal Terrae, Santander, 1999. 
superadoras, sino el modo de vivir ya como seguidores de Jesús y sus testigos, personalmente y como pueblo de Dios y comunidades cristianas.

En tercer lugar, decirnos todos que tenemos que huir del claustro, de la falta de pretensión evangélica, del discurso no performativo. La academia es beneficiosa e imprescindible, pero no podemos definirnos como académicos a dedicación exclusiva y ni siquiera a tiempo completo: tenemos que ser, ante todo, intelectuales y sentimentales orgánicos del pueblo de Dios y de los solidarios con él.

En cuarto lugar, que el binomio inextricable que forman Jesús de Nazaret y su Dios, y los pobres, debe ser el eje transversal que fecunde a la teología ${ }^{24}$.

Y finalmente, que al estar el Occidente y el no Occidente inextricablemente presentes en nuestra América, lo que hagan los occidentales americanos con los demás, si lo hacen como Dios manda, puede servir de estímulo a lo que habría que hacer en España. Esta tarea inexcusable entre nosotros acabará siéndolo también para los teólogos españoles.

24. J. Sobrino, Fuera de los pobres no hay salvación, Trotta, Madrid, 2007; P. Trigo, "Papel de la teología en el mundo actual", RLT, 73 (2008), pp. 67-71. 